\title{
Changes in Secondary Metabolites and Free Amino Acid Content in Tomato with Lamiaceae Herbs Companion Planting
}

\author{
Hasib Ahmad1, Manami Kobayashi², Yoh-ichi Matsubara ${ }^{2 *}$ \\ ${ }^{1}$ The United Graduate School of Agricultural Science, Gifu University, Gifu, Japan \\ ${ }^{2}$ Faculty of Applied Biological Sciences, Gifu University, Gifu, Japan \\ Email: *ymatsu@gifu-u.ac.jp
}

How to cite this paper: Ahmad, H., Kobayashi, M. and Matsubara, Y. (2020) Changes in Secondary Metabolites and Free Amino Acid Content in Tomato with $\mathrm{La}$ miaceae Herbs Companion Planting. American Journal of Plant Sciences, 11, 1878-1889. https://doi.org/10.4236/ajps.2020.1112134

Received: November 14, 2020

Accepted: December 4, 2020

Published: December 7, 2020

Copyright $\odot 2020$ by author(s) and Scientific Research Publishing Inc. This work is licensed under the Creative Commons Attribution International License (CC BY 4.0).

http://creativecommons.org/licenses/by/4.0/ (c) (i) Open Access

\begin{abstract}
The present experiment was conducted to evaluate the influence of Lamiaceae herbs companion planting on growth and secondary metabolites changes in tomato plants. Furthermore, the free amino acid changes in tomato due to companion planting were also evaluated using tomato-basil companionship as a model. Four-week-old seedlings of tomato were grown in a pot containing autoclaved commercial soil with basil, peppermint and hyssop as a companion plant separately in different density. Four weeks after companion planting, tomato plants under 1:1 companionship with the herbs showed significant increase in dry weights of shoots compared to control. Higher density of the herbs on the other hand expressed a growth suppression on tomato possibly due to nutrient competition. By the LC-MS analysis, shikimic acid and apigenin were identified as the major secondary compounds found in tomato plants and 1:1 companionship with basil seemed to have a positive influence on their content in tomato shoots. On the other hand, in case of peppermint and hyssop, the increase was observed in all parts of tomato plants. In addition, promotion in several free amino acid contents was also observed in tomato plants with basil companion planting compared to control. Thus, tomato plants grown with herb companion planting in 1:1 ratio seems to have a positive impact on growth of tomato. This positive influence might be related to the increase in some secondary metabolites and changes in the free amino acids observed in this study.
\end{abstract}

\section{Keywords}

Basil, Growth Promotion, Hyssop, Peppermint, Root Exudate

\section{Introduction}

Companion planting is an intercropping practice where two or more plants are 
grown together in a unit area. This practice of crop production is usually associated with organic agriculture especially in small scale farms or gardens which do not utilize much investment or technologies. The popularity of the companion planting method in garden production system is the result of popular books [1] [2], newspaper articles and internet websites. The target of companion planting is that, the plants involved will interact with each other agronomically and also influence the microclimate surrounding them [3] [4]. Besides influencing the microclimate, it is also used as a way of eco-friendly pest control method. That is, plants which directly mask the specific chemical cues are grown together to confuse the pests, or because they hold and retain particularly effective natural enemies of one another's pests [5]. However, certain disadvantages could arise between the companions due to competition for light, water and nutrients which may cause yield reduction of the main crop [6] [7] [8]. Still, garden producers have stood by their opinion that companion planting has the potential to be advantageous and to be adopted in conventional production. Adjustment of irrigation and fertilizer management [9], sowing companion crop with or after planting the main crop [10], selection of appropriate companion plant could possibly minimize the impact of competition in the companion planting system. Reports of increased yield in main crop under intercropped system through proper management are also present [6] [11].

One of the popular companion combinations mentioned by the gardeners is the tomato-basil companion planting [1]. It had been reported that basil acted as repellent of flies and improved the yield and taste of tomato when grown as companion [12]. Positive influence on control of insect pest like mites, mosquitos and tomato hornworm was also claimed by producers in tomato-basil companion system [2]. Research involving tomato-basil companion planting was reported to increase the total yield and biomass per plant compared to monoculture of tomato [4] [13]. However, the reason for this growth and yield increase phenomenon is still unclear. This positive phenomenon may not only involve pest control effect as claimed by gardeners, but also through some phytochemical changes in inner biochemical status of tomato due to the companionship. Furthermore, whether this phenomenon is exclusive to tomato-basil companionship or other Lamiaceae herbs shows the same effect is still unclear. As such, the present experiment was conducted to evaluate the impact of some Lamiaceae herbs companion planting on tomato growth and changes in the secondary metabolites. Furthermore, the free amino acid changes in tomato due to companion planting were also evaluated using tomato-basil companionship as a model.

\section{Materials and Methods}

\subsection{Growing of Tomato and Herb Seedlings and Setup of Companion System}

Seeds of basil (Ocimum basilicum L.), peppermint (Mentha piperita L.), hyssop (Hyssopus officinalis L.) and tomato (Solanum lycopersicum, cv. Momotaro 8) 
were used in the experiment. Tomato-basil companion planting was used as base model as it was the most common example of companion system given by the garden producers [1]. The other herbs were used to see whether this growth regulation effect was exclusive tomato-basil companion or other herbs of the same family could show similar results. Seeds of the herbs and tomato were sown in 72-hole seed tray $(280 \mathrm{~mm} \times 545 \mathrm{~mm} \times 49 \mathrm{~mm})$ filled with autoclaved commercial soil (Supermix A; Sakata Seed Corporation, Yokohama, Japan). Four weeks after sowing, the seedlings were transplanted to pots $(150 \mathrm{~mm} \times 130 \mathrm{~mm}, 1600$ $\mathrm{ml}$ ) also containing the autoclaved commercial soil. The experimental setups were: 1) Tomato mono culture (C), 2) Tomato + Basil (1:1), 3) Tomato + Basil $(1: 4), 4)$ Tomato + Basil $(1: 8), 5)$ Tomato + Basil with mesh (1:1 m), 6) Tomato + Basil with mesh (1:4 m), 7) Tomato + Basil with mesh (1:8 m), 8) Tomato + peppermint (1:1), 9) Tomato + peppermint (1:4), 10) Tomato + peppermint $(1: 8), 11)$ Tomato + Hyssop (1:1), 12) Tomato + Hyssop (1:4), 13) Tomato + Hyssop (1:8). The tomato-basil companion setup was used as a model for mesh separation treatment. A non-woven mesh was placed in the pot with the tomato plant in the center; separating tomato roots in all sides from the roots of basil plant/plants transplanted keeping a distance of $4 \mathrm{~cm}$. Ten pots per treatment were grown in greenhouse $25^{\circ} \mathrm{C} \pm 4^{\circ} \mathrm{C} / 20^{\circ} \mathrm{C} \pm 3^{\circ} \mathrm{C}$ day/night temperature with 12 - $13 \mathrm{~h}$ photoperiod $\left(750-1000 \mu \mathrm{mol} \cdot \mathrm{m}^{-2} \cdot \mathrm{s}^{-1}\right)$ and $60 \%-70 \%$ relative humidity.

\subsection{Growth Analysis of the Tomato Plants}

Four weeks after transplanting, the plants were uprooted and the roots of the tomato plants were cleaned from soil and debris. Then 4 tomato plants per treatment were taken and separated to shoot and root. The plant parts were dried using a constant temperature drier (ETTAS 600B; AS ONE Corporation, Osaka, Japan) at $80^{\circ} \mathrm{C}$ for two days. Then the dry weight of shoots and roots were measured. The rest of the plants were cryopreserved for further analysis. Based on the results of the growth parameter considered, the tomato plants under 1:1 treatment were considered for secondary metabolites analysis.

\subsection{Analysis of Secondary Metabolites in Tomato}

Analysis of secondary metabolites in tomato was conducted according to the method of reference [14]. From the cryopreserved samples of five plants, $1.2 \mathrm{~g}$ of tomato plant parts (leaf, stem and root) were pulverized separately in mortar with liquid nitrogen to give a fine powder and mixed with $6 \mathrm{ml}$ ultrapure water to prepare sample extract solutions. The sample solutions were then centrifuged $\left(13,000 \mathrm{rpm}, 4^{\circ} \mathrm{C}, 15 \mathrm{~min}\right)$ and the supernatant was filtered through a sterilizing filter $(0.45 \mu \mathrm{m}$; ADVANTECH Co. Ltd., Japan). The samples were further centrifuged (13,000 rpm, $4^{\circ} \mathrm{C}, 15 \mathrm{~min}$ ) using Nanosep $10 \mathrm{~K}$ (Nihon Pall Ltd. Tokyo, Japan) to remove proteins in the extract.

The samples were analyzed using UPLC-MS/MS (Waters Corporation, Mil- 
ford, USA). A reversed-phase column (ACQUITY UPLC BEH C18, $1.7 \mu \mathrm{m}, 2.1$ $\times 100 \mathrm{~mm}$; Waters Corporation, Milford, USA) with a thermostation at $40^{\circ} \mathrm{C}$ was used for the analysis. The mobile phases comprised $0.1 \%$ formic acid in water (A) and acetonitrile (B) at a flow rate of $0.4 \mathrm{~mL} / \mathrm{min}$. The sample injection volume was $7.5 \mu \mathrm{L}$. The gradient profile was as follows: 0 - $6 \mathrm{~min}, 95 \% \mathrm{~A} ; 6$ - 12 min, 75\% A; 12 - 30 min, 65\% A; 30 - $32.5 \mathrm{~min}, 5 \% \mathrm{~A}$; and 32.5 - $35 \mathrm{~min}, 95 \% \mathrm{~A}$. The mass range of electrospray ionization was analyzed in negative mode at 50 $1000 \mathrm{~m} / \mathrm{z}$ using a mass spectrometer (Xevo Q Tof MS, Waters Corporation, Milford, USA), and the MS/MS collision was performed at $30 \mathrm{~V}$. A mass chromatogram of the $m / z$ value of each component in the extract was prepared from the measurements obtained using the retention time.

\subsection{Analysis of Free Amino Acid Content in Tomato}

The tomato plants under 1:1 treatment with basil were selected as a model to evaluate the free amino acid content change due to companion planting according to the method of reference [15]. Cryopreserved samples $(0.2 \mathrm{~g}$ of leaves, stems and roots each) were extracted using $3 \mathrm{ml}$ of $0.2 \mathrm{~N}$ perchloric acid solution and then centrifuged at $13,000 \mathrm{rpm}$ at $4^{\circ} \mathrm{C}$ for $5 \mathrm{~min}$. The supernatant was subsequently filtered through a syringe filter (GL Chromatodisc, GL Sciences Co., Ltd.) for use as an analysis sample.

Analytical samples were derivatized using the AccQ.Tag Ultra Derivatization Kit (Waters Corporation, Milford, USA). Analytical samples $(30 \mu \mathrm{L})$ were mixed with $210 \mu \mathrm{Lof}$ borate buffer and $60 \mu \mathrm{L}$ of derivatization reagent. The reaction mixture was immediately mixed and left for $1 \mathrm{~min}$ at room temperature. Subsequently, the solution was incubated at $55^{\circ} \mathrm{C}$ for $10 \mathrm{~min}$ in water bath. After cooling, the reaction mixture was used for UPLC injection.

The samples were analyzed using UPLC-MS/MS (Waters Corporation, Milford, USA). A reversed-phase column (ACQUITY UPLC BEH C18, $1.7 \mu \mathrm{m}, 2.1$ $\times 100 \mathrm{~mm}$; Waters Corporation, Milford, USA) with a thermostation at $40^{\circ} \mathrm{C}$ was used for the analysis. The mobile phases comprised $0.1 \%$ formic acid in water (A) and acetonitrile (B) at a flow rate of $0.4 \mathrm{~mL} / \mathrm{min}$. A sample injection volume of $5 \mu \mathrm{L}$ was used for the analysis. The gradient profile was as follows: initial, $0.1 \% \mathrm{~B} ; 0-12 \mathrm{~min}, 50 \% \mathrm{~B} ; 12-13 \mathrm{~min}, 0.1 \% \mathrm{~B}$ and $13-15 \mathrm{~min}, 0.1 \% \mathrm{~B}$. The mass range of electrospray ionization was analyzed in positive mode at 100 $1000 \mathrm{~m} / \mathrm{z}$ using a mass spectrometer (Xevo Q Tof MS, Waters Corporation, Milford, USA). A mass chromatogram (Abs Window $0.05 \mathrm{Da}$ ) of the $\mathrm{m} / z$ value of each amino acid content was prepared for the measurement result and the amino acid content was measured according to peak integration value. Amino acid mixed standard solution H-type (Wako Pure Chemical Industries Ltd. Japan) was used for making a standard curve. Twenty-one types of free amino acids were analyzed, all of which were included in the standard solution.

\subsection{Statistical Analysis}

The mean values for dry weight of shoots and roots of tomato were analyzed by 
Tukey's multiple range test at $\mathrm{P}<0.05$. For free amino acid content, the significant differences were determined based on $t$ test at $\mathrm{P}<0.05$. All the analyses were conducted using XLSTAT 2012 pro statistical analysis software (Addinsoft Inc. New York, USA).

\section{Results}

Four weeks after transplanting, the dry weights of tomato shoots and roots under companion planting with basil, peppermint and hyssop showed varying degree of change compared to control (Figure 1 and Figure 2). The highest dry weights in shoots were observed in plants under 1:1 companion treatment compared to control in all the cases. Even in the case of mesh separation in tomato-basil companion system, the highest dry weight of shoots was observed in 1:1 combination which also showed statistical similarity 1:1 without mesh. The dry weights of roots except for hyssop companionship, did not show any significant difference with control. However, growth restriction was observed in the higher density companion treatments especially in 1:8 treatment. In these cases, the dry wights were found to be lower than that of the plants under control except in peppermint.

From the analysis of secondary metabolites in tomato plants, the major compounds that were identified in tomato plants were shikimic acid and apigenin. The content of both these compounds was found to have increased in tomato stem when grown in association with basil (Table 1). However, no significant increase was observed in case of leaves and roots of tomato plants under basil companion planting. On the other hand, in both peppermint and hyssop companionship, the content of both shikimic acid and apigenin increased in all parts of tomato plants.
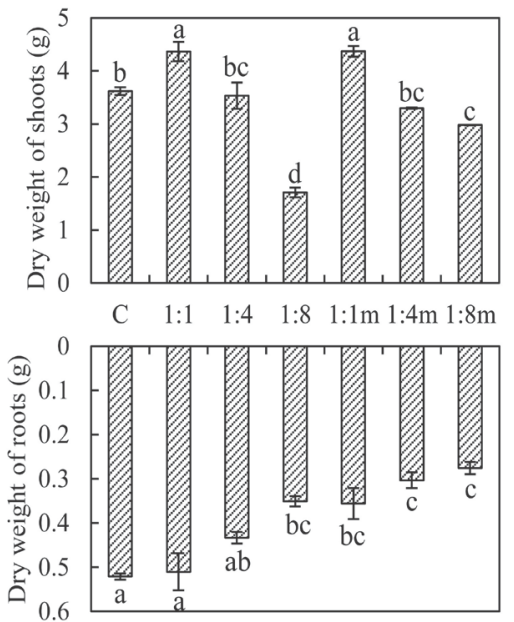

Figure 1. Dry weight of shoots and roots of tomato plants with basil companion planting. C, single tomato; $1: 1$, tomato + basil $(1: 1) ; 1: 4$; tomato + basil $(1: 4) ; 1: 8$, tomato + basil (1:8); 1:1 m, tomato + basil with mesh (1:1), 1:4 m, tomato + basil with mesh (1:4); 1:8 m, tomato + basil with mesh $(1: 8)$. Columns denoted by different letters indicate significant difference according to Tukey's test $(\mathrm{P}<0.05)$. 

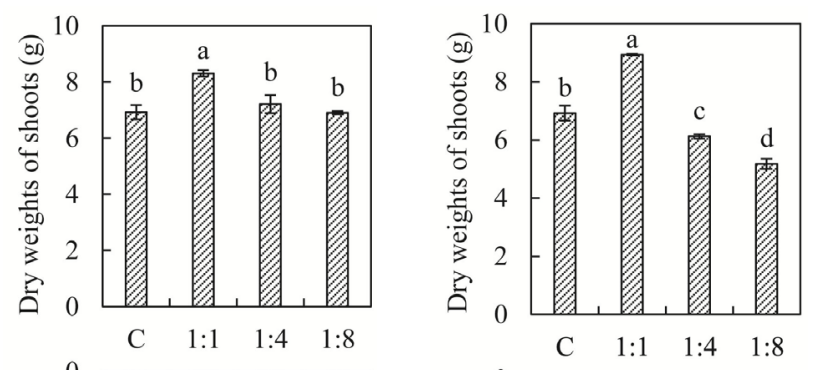

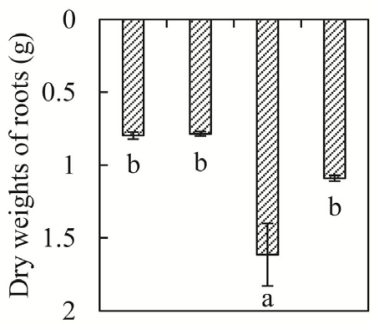

(a)

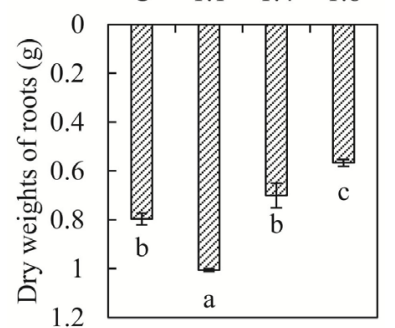

(b)

Figure 2. Dry weight of shoots and roots of tomato plants with peppermint (a) and hyssop (b) companion planting. C, single tomato; $1: 1$, tomato + peppermint/hyssop (1:1); 1:4; tomato + peppermint/hyssop (1:4); 1:8, tomato + peppermint/hyssop (1:8). Columns denoted by different letters indicate significant difference according to Tukey's test $(\mathrm{P}<$ $0.05)$.

Table 1. Influence of companion plants on changes in secondary metabolite content in tomato.

\begin{tabular}{|c|c|c|c|}
\hline Companion plant & Tomato plant part & Shikimic acid & Apigenin \\
\hline \multirow{3}{*}{ Basil } & Leaves & - & - \\
\hline & Stem & O & O \\
\hline & Roots & - & - \\
\hline \multirow{3}{*}{ Peppermint } & Leaves & O & O \\
\hline & Stem & O & O \\
\hline & Roots & $\bigcirc$ & O \\
\hline \multirow{3}{*}{ Hyssop } & Leaves & O & O \\
\hline & Stem & O & O \\
\hline & Roots & 0 & 0 \\
\hline
\end{tabular}

Here, -, no change; $\bigcirc$, increased.

Regarding the changes in free amino acid contents of tomato due to basil companionship, several amino acid contents changed significantly in leaves and shoots of plants under 1:1 treatment compared to control. In case of leaves, significant increase was observed in phenyl-alanine, iso-leucine, valine, alanine, lysine and GABA contents in plants under 1:1 treatment compared to control (Figure 3). In contrast, higher content of valine, alanine, proline, GABA, serine and glutamine was observed in the stems of plants under 1:1 companion treatment compared to control (Figure 4). In both cases, the amino acids other than 

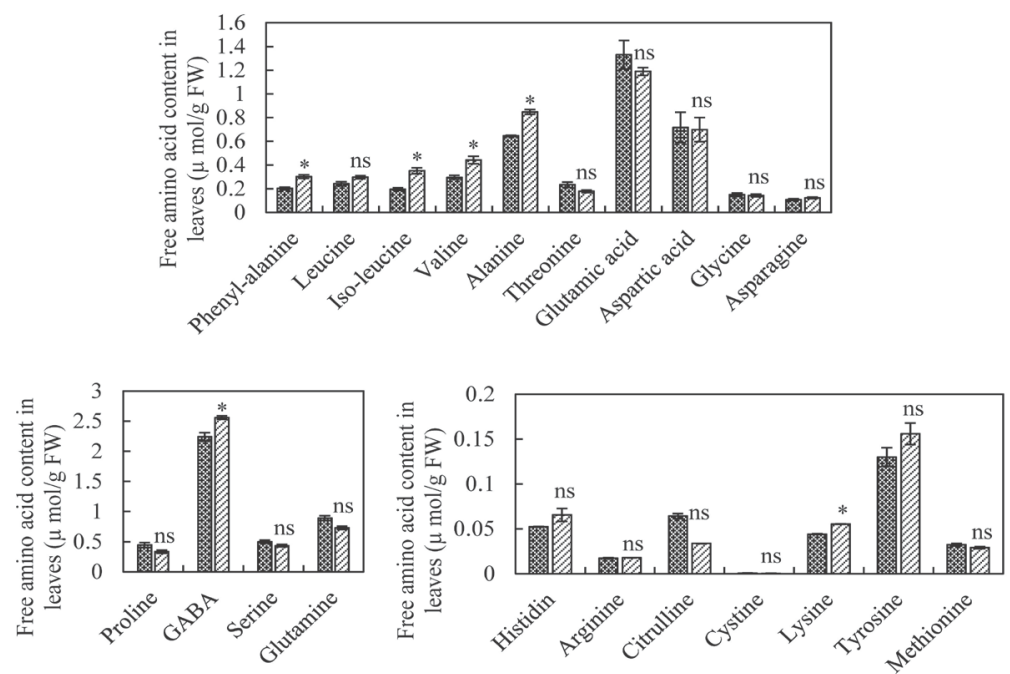

Figure 3. Free amino acid content in leaves of tomato plants grown with basil companion planting. , Tomato mono; $\mathbb{Z}$, Tomato + Basil (1:1). ${ }^{\star}$, significantly different according to $t$-test $(\mathrm{P}<0.05)$; ns, non-significant.
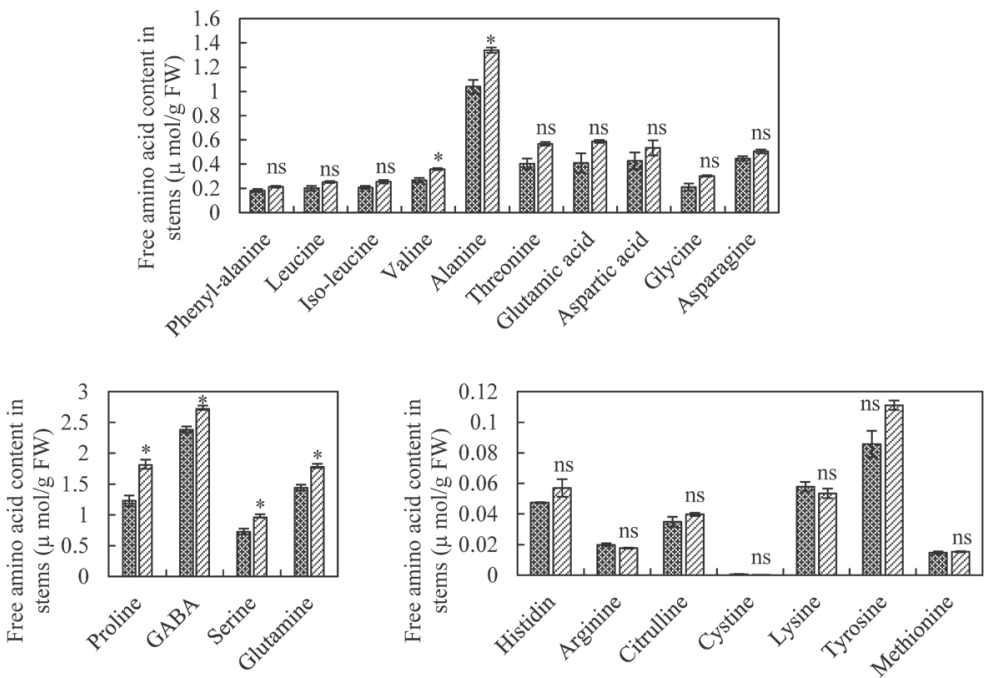

Figure 4. Free amino acid content in stems of tomato plants grown with basil companion planting. 図, Tomato mono; $\square$, Tomato + Basil (1:1). ${ }^{\star}$, significantly different according to $t$-test $(\mathrm{P}<0.05)$; ns, non-significant.

the ones mentioned in leaves and shoots respectively did not express any significant increase or decrease. However, in case of roots, no significant difference was observed in any free amino acid contents between the treatment considered and control (data not shown).

\section{Discussion}

In the present study, tomato plants grown with basil companionship showed a varying degree of growth expression depending on the plant density. The 1:1 combination appeared to have growth boosting ability compared to control. This may be attributed to the less competitive behavior from basil in the companion 
system as reported by [4]. According to the report, basil plants tend to be subordinate crop in companion crop system. This although resulting in growth retardation of basil, does not interfere with the growth of the major or dominant crop. As such, the competition between tomato and basil plants in 1:1 system for nutrition, water, light and space was lower that contributed to the growth of tomato plants. Furthermore, this positive phenomenon of companionship on tomato is not limited to only basil as observed from the present study. Herbs of Lamiaceae family like peppermint and hyssop seemed to have the similar growth promoting influence on tomato as evident from the results. However, with the higher density of the herb plants like 1:4 or 1:8, the competition pressure accumulated and thus hampered the growth of tomato plants as observed in the result. Trials with other crops under companion planting system also exhibited better growth with efficient use of the net input available, turning up better yields [16]. Reference [17] had also found similar growth and yield improvement in crops grown together than individually and attributed these phenomena to better utilization of available resources by the plants.

The major components identified in tomato plants through UPLC-MS analysis were shikimic acid and apigenin, and their content in tomato shoot was influenced significantly in plants grown with herb companionship. It has been reported that, intercropping pressure has the ability to influence the accumulation of minerals and other secondary metabolites in plants increasing their quality [18] [19] [20]. Similar phenomenon must have occurred in the present experiment. The growth regulation in tomato plants by shikimic acid was reported to be mediated through increasing longevity of leaves by retaining chlorophylls and increasing mineral contents [21]. Furthermore, shikimic acid has been related to the increased contents of soluble sugar and lycopene which influences the taste and appearance of tomato [21] [22]. This could possibly explain the claim of the garden producers about the improvement of taste of tomato grown in herb companionship. On the other hand, flavonoids like apigenin play several important roles in plant growth and development like increasing tolerance to biotic and abiotic stress, hormone transportation, acting as phytoalexins etc. As such, an increase in these compounds is desirable in plants to maintain as well as boost the growth and subsequently the yield and quality of a crop.

Regarding free amino acids, a significant increase was observed in several of them in both leaves and stems of tomato plants. The influence of basil companion planting on increased shikimic acid production may have played a role in the increase in several amino acids; as shikimate pathway is used by plants for biosynthesis of aromatic amino acids [23]. Amino acids play several roles in plant life such as stress management, hormone precursors and regulation [24] [25]. Besides these, another important role played by the amino acids is the regulation of several physiological processes in plants like nutrient uptake specially nitrogen, antioxidant metabolism and root development [26] [27] [28]. Amino acids like phenylalanine, L-alanine, leucine, lysine and glutamine had been reported to influence glutamate receptors in plants [29] that in turn mediate a 
number of plant responses like changes in root architecture, plant stress signaling, carbon metabolism, stomatal movements, photosynthesis and plant immunity [27]. GABA has been reported to act as endogenous signaling molecule in plants that regulates growth and development and protect from various environmental stresses [30]. Proline had been reported to influence in plant growth and development by being an important component of cell wall matrix to combat stress conditions [31] whereas serine had been reported to participate in the biosynthesis of several biomolecules required for cell proliferation, including amino acids, nitrogenous bases, phospholipids, and sphingolipids [32]. So, an increase in these important free amino acids in tomato plants possibly played an important role in the growth improvement observed from the experiment.

In the present experiment, the soil used for growing both tomato and herb plants was autoclaved prior seed sowing and subsequent transplanting. This eliminated the possibility of any microorganism influence on the growth improvement in tomato. Furthermore, in the case of mesh separation, no physical contact between the tomato and basil roots was present. As such, no physical stimulation or signaling could be present. So, the only possible way for any influence occurring from the companion planting system was through chemicals ques generated from the herb companions. To further evaluate this hypothesis, the relative growth of the basil, peppermint and hyssop compared to tomato (by visual observation), index of tomato shoot dry weights and aroma (by smell test) were measured (Table 2). From the evaluation it was observed that relative to tomato, the growth of basil, peppermint and hyssop could be graded as high, medium and low respectively. Furthermore, in aroma evaluation, both basil and peppermint gave distinguishable aromatic presence whereas hyssop had a very low aromatic presence. However, the tomato plants under hyssop companionship showed higher shoot dry weight index compared to basil or peppermint. If we consider the volatile compound or essential oils of the herbs to be responsible for growth improvement in tomato, then plants with hyssop companionship should express a lower dry weight index compared to basil and peppermint. As such, the only possible explanation of the growth improvement observed in tomato would be the influence of some root exudates from the herbs. However, further in-depth evaluation is necessary to clarify it. Identification of such biochemical influencers would enable us to gain the beneficial effect with their

Table 2. Influence of companion planting on herb growth, tomato dry weight indices and aroma quality of the herbs.

\begin{tabular}{cccc}
\hline Parameters & Basil & Peppermint & Hyssop \\
\hline Herb growth & High & Medium & Low \\
Index of tomato shoot dry weights ${ }^{\mathrm{a}}$ & 120 & 119 & 129 \\
Aroma quality & High & High & Low \\
\hline
\end{tabular}

a. Calculated as: Shoot dry weight index $=\frac{\text { Dry weight of plants under } 1: 1 \text { companionship }}{\text { Dry weight of plants in mono culture }} \times 100$. 
direct application in the crop production system and open a new avenue of crop growth promotion.

\section{Conclusion}

The present experiment evaluated the growth improvement and changes in secondary metabolites and amino acids in tomato when grown in companion with Lamiaceae herbs. The results indicated a growth improvement in 1:1 combination of tomato and herbs with increase in some important secondary metabolites and amino acids. Increase of these primary and secondary molecules might have played an important role in the growth promotion. The present experiment acts as a fundamental research to address the longstanding claim from garden producers about the beneficial impact of such companionship. However, further in-depth analysis is required to clarify the mechanisms involved in the growth and subsequent yield improvement of tomato plants.

\section{Acknowledgements}

This study was supported by Grants-in-Aid for Scientific Research (No. 20K06033), Japan Society for the Promotion of Science (JSPS).

\section{Conflicts of Interest}

The authors declare no conflicts of interest regarding the publication of this paper.

\section{References}

[1] Riotte, L. (1975) Carrots Love Tomatoes: Secrets of Companion Planting for Successful Gardening. Storey Books, Pownal, Vermont.

[2] Cunningham, S.J. (1998) Great Garden Companions: A Companion-Planting System for a Beautiful, Chemical-Free Vegetable Garden. Rodale Press, Emmaus, Pennsylvania.

[3] Gómez-Rodríguez, O., Zavaleta-Mejía, E., González-Hernández, V.A., Livera-Muñoz, M. and Cárdenas-Soriano, E. (2003) Allelopathy and Microclimatic Modification of Intercropping with Marigold on Tomato Early Blight Disease Development. Field Crops Research, 83, 27-34. https://doi.org/10.1016/S0378-4290(03)00053-4

[4] Bomford, M.K. (2009) Do Tomatoes Love Basil but Hate Brussels Sprouts? Competition and Land-Use Efficiency of Popularly Recommended and Discouraged Crop Mixtures in Biointensive Agriculture Systems. Journal of Sustainable Agriculture, 33, 396-417. https://doi.org/10.1080/10440040902835001

[5] Parker, J.E., Snyder, W.E., Hamilton, G.C. and Rodriguez-Saona, C. (2013) Companion Planting and Insect Pest Control. Weed and Pest Control-Conventional and New Challenges. IntechOpen, London, UK.

[6] Lu, Y., Watkins, K.B., Teasdale, J.R., Aref, A. and Abdul-Baki, A. (2000) Cover Crops in Sustainable Food Production. Food Reviews International, 16, 121-157. https://doi.org/10.1081/FRI-100100285

[7] Jedrszczyk, E. and Poniedziałek, M. (2007) The Impact of the Living Mulch on Plant Growth and Selected Features of Sweet Corn Yield. Folia Horticulturae, 19, 3-13. 
[8] Borowy, A. (2012) Growth and Yield of Stake Tomato under No-Tillage Cultivation Using Hairy Vetch as a Living Mulch. Acta Scientiarum Polonorum Hortorum, 11, 229-252.

[9] Kołota, E. and Adamczewska-Sowińska, K. (2013) Living Mulches in Vegetable Crops Production: Perspectives and Limitations (A Review). Acta Scientiarum Polonorum Hortorum, 12, 127-142.

[10] Adamczewska-Sowińska, K. and Kołota, E. (2008) The Effect of Living Mulches on Yield and Quality of Tomato Fruits. Vegetable Crops Research Bulletin, 69, 31-38.

[11] Mandal, S.M.A. and Dash, D. (2012) Effect of Intercropping on the Incidence of Insect Pests and Yield in Cabbage. Journal of Plant Protection and Environment, 9, 26-28.

[12] Bradley, F.M. and Ellis, B.W. (1992) All-New Encyclopedia of Organic Gardening. Rodale Press, Emmaus, Pennsylvania.

[13] de Carvalho, L.M., de Oliveira, I.R., Almeida, N.A. and Andrade, K.R. (2012) The Effects of Biotic Interaction between Tomato and Companion Plants on Yield. Acta Horticulturae, 933, 347-354. https://doi.org/10.17660/ActaHortic.2012.933.45

[14] De Vos, R., Moco, S., Lommen, A., Keurentjes, J.J.B., Bino, R.J. and Hall, R.D. (2007) Untargeted Large-Scale Plant Metabolomics Using Liquid Chromatography Coupled to Mass Spectrometry. Nature Protocols, 2, 778-791.

https://doi.org/10.1038/nprot.2007.95

[15] Nimbalkar, M.S., Pai, S.R., Pawar, N.V., Oulkar, D. and Dixit, G.B. (2012) Free Amino Acid Profiling in Grain Amaranth Using LC-MS/MS. Food Chemistry, 134, 2565-2569. https://doi.org/10.1016/j.foodchem.2012.04.057

[16] Feike, T., Chen, Q., Graeff-Honninger, S., Pfenning, J. and Claupein, W. (2010) Farmer-Developed Vegetable Intercropping Systems in Southern Hebei, China. Renewable Agriculture and Food Systems, 25, 272-280.

https://doi.org/10.1017/S1742170510000293

[17] Miyazawa, K., Murakami, T., Takeda, M. and Murayama, T. (2010) Intercropping Green Manure Crops-Effects on Rooting Patterns. Plant and Soil, 331, 231-239. https://doi.org/10.1007/s11104-009-0248-y

[18] Inal, A., Gunes, A., Zhang, F. and Cakmak, I. (2007) Peanut/Maize Intercropping Induced Changes in Rhizosphere and Nutrient Concentrations in Shoots. Plant Physiology and Biochemistry, 45, 350-356.

https://doi.org/10.1016/j.plaphy.2007.03.016

[19] Li, L., Tilman, D., Lambers, H. and Zhang, F.S. (2014) Plant Diversity and Overyielding: Insights from Belowground Facilitation of Intercropping in Agriculture. New Phytologist, 203, 63-69. https://doi.org/10.1111/nph.12778

[20] Tong, Y., Gabriel-Neumann, E., Krumbein, A., Ngwene, B., George, E. and Schreiner, M. (2015) Interactive Effects of Arbuscular Mycorrhizal Fungi and Intercropping with Sesame (Sesamum indicum) on the Glucosinolate Profile in Broccoli (Brassica oleracea var. Italica). Environmental and Experimental Botany, 109, 288-295. https://doi.org/10.1016/j.envexpbot.2014.06.008

[21] Al-Amri, S.M. (2013) Improved Growth, Productivity and Quality of Tomato (Solanum lycopersicum L.) Plants through Application of Shikimic Acid. Saudi Journal of Biological Sciences, 20, 339-345. https://doi.org/10.1016/j.sjbs.2013.03.002

[22] Favati, F., Lovelli, S., Galgano, F., Miccolis, V., Di Tommaso, T. and Candido, V. (2009) Processing Tomato Quality as Affected by Irrigation Scheduling. Scientia Horticulturae, 122, 562-571. https://doi.org/10.1016/j.scienta.2009.06.026 
[23] Santos-Sánchez, N.F., Salas-Coronado, R., Hernández-Carlos, B. and Villanueva-Cañongo, C. (2019) Shikimic Acid Pathway in Biosynthesis of Phenolic Compounds. In: Plant Physiological Aspects of Phenolic Compounds, IntechOpen, London, UK.

[24] Zhao, Y. (2010) Auxin Biosynthesis and Its Role in Plant Development. Annual Review of Plant Biology, 61, 49-64.

https://doi.org/10.1146/annurev-arplant-042809-112308

[25] Maeda, H. and Dudareva, N. (2012) The Shikimate Pathway and Aromatic Amino Acids Biosynthesis in Plants. Annual Review of Plant Biology, 63, 73-105. https://doi.org/10.1146/annurev-arplant-042811-105439

[26] Miller, A.J., Fan, X., Shen, Q. and Smith, S.J. (2007) Amino Acids and Nitrate as Signals for the Regulation of Nitrogen Acquisition. Journal of Experimental Botany, 59, 111-119. https://doi.org/10.1093/jxb/erm208

[27] Weiland, M., Mancuso, S. and Baluska, F. (2015) Signalling via Glutamate and GLRs in Arabidopsis thaliana. Functional Plant Biology, 43, 1-25. https://doi.org/10.1071/FP15109

[28] Hildebrandt, T.M., Nunes Nesi, A., Araújo, W.L. and Braun, H.P. (2015) Amino Acid Catabolism in Plants. Molecular Plant, 8, 1563-1579. https://doi.org/10.1016/j.molp.2015.09.005

[29] Forde, B.G. and Roberts, M.R. (2014) Glutamate Receptor-Like Channels in Plants: A Role as Amino Acid Sensors in Plant Defence? F1000Prime Reports, 6, 37.

[30] Ramos-Ruiz, R., Martinez, F. and Knauf-Beiter, G. (2019) The Effects of GABA in Plants. Cogent Food and Agriculture, 5, Article ID: 1670553. https://doi.org/10.1080/23311932.2019.1670553

[31] Kishor, P.B.K. and Sreenivasulu, N. (2014) Is Proline Accumulation per se Correlated with Stress Tolerance or Is Proline Homoeostasis a More Critical Issue? Plant, Cell and Environment, 37, 300-311. https://doi.org/10.1111/pce.12157

[32] Ros, R., Muñoz-Bertomeu, J. and Krueger, S. (2014) Serine in Plants: Biosynthesis, Metabolism, and Functions. Trends in Plant Science, 19, 564-569. https://doi.org/10.1016/j.tplants.2014.06.003 